\section{ORIGINAL RESEARCH}

\author{
R.V. Chandra \\ C.P. Law \\ B. Yan \\ R.J. Dowling \\ P.J. Mitchell
}

\title{
Glasgow Coma Scale Does Not Predict Outcome Post-Intra-Arterial Treatment for Basilar Artery Thrombosis
}

BACKGROUND AND PURPOSE: Evidence to guide patient selection for IA therapy in acute basilar artery thrombosis is lacking. The GCS is frequently used as a selection criterion, based on the view that higher GCS correlates with better neurologic outcome. This view has not been systematically studied. We hypothesize that low GCS does not correlate with poor outcome and that it should not preclude IA therapy.

MATERIALS AND METHODS: We included 40 consecutive patients with basilar artery thrombosis treated with IA therapy. Clinical characteristics, GCS, time to intervention, and postprocedural TIMI scores were collected. Recanalization was defined as TIMI grade 2 or 3; clinical outcome was measured by 90-day mRS.

RESULTS: Median patient age was 63.5 years. Most presented with gaze palsies (67.5\%) or hemiparesis (45\%). Median GCS was 9, the median time to intervention was 7.2 hours, and recanalization rate was $82.5 \%$. Good neurologic outcome (mRS $\leq 1$ ) occurred in $30 \%$. There was no correlation between GCS and 90-day mRS (Spearman $\rho-0.174, P=.283$ ). Equal numbers of patients with good neurologic outcome of $\mathrm{mRS} \leq 1(n=12)$ had a GCS of $\leq 6$ or $>6$. In those with GCS $\leq 6$ for $>3$ hours, 33.3\% had good neurologic outcome-a similar rate to that of the overall cohort. Statistical significance was demonstrated between time to IA therapy within 6 hours and mRS $\leq 2$.

CONCLUSIONS: Low GCS score did not correlate with poor neurologic outcome in patients with acute basilar artery thrombosis managed with IA therapy. It is not appropriate to exclude patients from IA therapy on the basis of low GCS.

ABBREVIATIONS: $\mathrm{ACT}=$ activated clotting time; $\mathrm{AICA}=$ anterior inferior cerebellar artery; $\mathrm{Cl}=$ confidence interval; GCS = Glasgow Coma Scale; $I A=$ intra-arterial; IQR = interquartile range: $\mathrm{mRS}=$ modified Rankin Scale; SCA = superior cerebellar artery; TIMI = Thrombolysis In Myocardial Infarction; $\mathrm{VA}=$ vertebral artery

A cute basilar artery thrombosis is an infrequent but catastrophic subtype of posterior circulation ischemic stroke that carries a mortality rate of $80 \%-90 \%$ without treatment. ${ }^{1-4}$ Consequent thalamic, brain stem, and cerebellar infarction can result in herniation, hydrocephalus, and death. Achieving timely vascular recanalization is the aim of treatment ${ }^{5}$; however, evidence guiding appropriate patient selection for recanalization is lacking, in particular for IA recanalization.

The spontaneous recanalization rate is unclear but is likely poor, ${ }^{6}$ and Lindsberg and Mattle ${ }^{7}$ have suggested that death and dependency rates in those treated with intravenous thrombolysis are similar to those treated with IA therapies. Only 1 multicenter randomized controlled trial assessed IA therapy efficacy for acute basilar thrombosis. The Australian Urokinase Stroke Trial ${ }^{8,9}$ recruited 16 patients during 7 years and found that good neurologic outcomes were more frequent

Received June 4, 2010; accepted after revision July 26

From the Departments of Radiology (R.V.C., C.P.L., R.J.D., P.J.M.) and Neurology (B.Y.) and Comprehensive Stroke Centre and Neurointervention Service (R.J.D., P.J.M., B.Y.), The Royal Melbourne Hospital, Melbourne, Australia

Peter Mitchell, Richard Dowling, and Bernard Yan received research support for participation in the Interventional Management of Stroke Trial III.

Paper previously presented at: Annual Meeting of the American Society of Neuroradiology, May 15-20, 2010; Boston, Massachusetts.

Please address correspondence to Peter Mitchell, MBBS, MMed, Neurointervention Service, Department of Radiology, Royal Melbourne Hospital, Parkville, VIC 3050, Australia; e-mail: Peter.Mitchell@mh.org.au

DOI 10.3174/ajnr.A2325

in the IA lysis treatment group (50\%) compared with the placebo control group (12.5\%). ${ }^{9}$

Thus, current management algorithms are largely based on observational studies, mainly retrospective, and meta-analyses that pool individual study data. These have revealed that IA thrombolysis results in significantly higher recanalization rates, ${ }^{6,710}$ and that recanalization is independently associated with reduced mortality and better functional outcome. . $^{3-9,11-14}$ Presenting clinical status, GCS score, age, and time to intervention influence the decision to proceed with IA therapy. ${ }^{5,15}$ There is little evidence to guide this decision because reported studies have had variable exclusion criteria (eg, tetraplegia for $>6$ hours, bilateral mydriasis and extinct brain stem reflexes for $>1$ hour, and extensive infarction on baseline imaging ${ }^{11}$; GCS score $<9$ for $>6$ hours $^{9}$; symptom onset of $>12$ hours $^{12}$; and coma for $>4$ hours $^{3}$ ).

The GCS is frequently used as a selection criterion for IA treatment, with the view that high GCS is associated with better neurologic outcome. ${ }^{11,16,17}$ However, Tsao et al, ${ }^{17}$ who state that initial GCS predicts outcome following thrombolysis in posterior circulation stroke, had a total of 21 patients, of whom only 12 received IA therapy (9, urokinase; 3 , IA tissue plasminogen activator). In addition, the patients of Tsao et al were treated without the use of mechanical thrombectomy, angioplasty, or stent insertion, so this small cohort may not be applicable to current patient cohorts with newer methods of revascularization. Ezaki et $\mathrm{al}^{16}$ used a GCS of 10 as an arbitrary 
cutoff in assessing outcome, which does not acknowledge significant clinical differences from GCS 3-9.

We hypothesize that a low GCS of $\leq 6$ does not correlate with poorer neurologic outcome after IA treatment in acute basilar thrombosis and propose that low GCS should not preclude IA treatment.

\section{Materials and Methods}

A retrospective single-center review was conducted of 40 consecutive patients with angiographically confirmed complete basilar artery thrombosis and immediate IA intervention between 1993 and 2009. Our comprehensive stroke referral service has 24-hour neurointerventional support, with treatment offered in the study population $\leq 30$ hours post-symptom onset. The only exclusion criterion was extensive infarction on preoperative CT with no limiting criteria for age or GCS.

Clinical parameters were identified through medical record review. "GCS" was defined at the lowest GCS score documented before IA therapy. "Time to IA intervention" was defined from the time of symptom onset, or from the time when the patient was last seen neurologically well, to the time of treatment onset. In the subgroup of patients with a variable clinical course followed by sudden onset of coma, time was defined from the sudden deterioration in clinical state.

Angiograms were reviewed by 2 neurointerventionists, with consensus opinion reached on clot location, collateral supply, and postprocedural TIMI score. Clot location was modeled on the methodology of Archer and Horenstein ${ }^{1}$ — proximal basilar (basilar origin to the origins of AICAs), midbasilar (origins of AICAs to origins of the SCA), and top of the basilar (origins of the SCAs to the basilar bifurcation). Involvement of the intra- or extradural VAs was also recorded; if either was involved bilaterally, this involvement was noted. Collateral supply from the posterior inferior cerebellar arteries via surface collaterals to the SCA territory and from the posterior communicating arteries to the basilar artery (when selective carotid angiography was performed) was also recorded.

TIMI was classified as grade 0 , complete occlusion or no flow/ perfusion of distal vasculature; grade 1, minimal flow/perfusion but with no opacification of distal vasculature; grade 2, partial obstruction leading to delayed opacification of distal vasculature; and grade 3 , complete unimpeded perfusion of the distal vasculature regardless of whether residual stenosis was present. ${ }^{16}$ "Recanalization" was defined as TIMI grade 2 or 3 .

After diagnostic angiography confirmed basilar artery thrombosis and intrathrombus placement of the microcatheter was achieved, an initial 200,000 U of urokinase with $5000 \mathrm{U}$ of heparin was administered, followed by pulsed hand injections of further urokinase ( $m a x-$ imum 1,000,000 U). Heparin bolus was administered according to weight with $70 \mathrm{U} / \mathrm{kg}$ to a maximum of $5000 \mathrm{U}$, followed by baseline and hourly ACT monitoring aiming at an ACT of 250. Total periods of thrombolysis were 1-2 hours, with repeat selective angiography every 15 minutes to assess recanalization. If flow disruption or occlusion persisted, angioplasty (percutaneous transarterial angioplasty) and/or stent placement was performed, particularly in atherothrombotic occlusions. If a full dose of intravenous thrombolysis had been administered, we proceeded to mechanical thrombectomy with the Merci retriever (Concentric Medical, Mountain View, California). Postoperatively, heparin infusion was started at the end of the procedure when, in the opinion of the interventionist, rethrombosis or ongoing thromboembolism was a major risk, exceeding the estimated risk of hemorrhagic complication. This was monitored with an activated partial thromboplastin time of $60-65$ as the target range, which was continued for 24 hours but was stopped if there was evidence of significant infarction or hemorrhage. If the underlying vessels were completely normal following lysis, confirming a diagnosis of embolus, heparin could be stopped at the end of the procedure.

Clinical outcome was assessed with 90-day mRS score obtained from the medical records. Primary analysis defined good neurologic outcome as $\mathrm{mRS} \leq 1$, as stratified by Demchuk et al. ${ }^{18}$ A secondary analysis by using $\mathrm{mRS} \leq 2$ as a good neurologic outcome was also performed for further comparison with the existing literature. ${ }^{4,14,17}$

Statistical assessment was performed by the Clinical Epidemiology and Health Service Evaluation Unit (Royal Melbourne Hospital, Parkville, Australia). Data analysis was performed by using STATA10 (StataCorp, College Station, Texas)) with a $P$ value $<.05$ considered statistically significant. The Wilcoxon rank sum test was used to determine the differences between ordinal variables (eg, age), and the Spearman correlation was used to find the correlation between GCS and 90-day mRS scores. The Fisher exact test was used to determine the correlation between categoric variables. Stepwise logistic regression was used to find predictors of good neurologic outcome. Study approval was granted by the local research and ethics committee.

\section{Results}

Of the 40 patients, $62.5 \%$ were men. Median age was 63.5 years with an IQR of 47-77 years. The clinical presentation included gaze palsies $(68 \%)$, hemiparesis (45\%), quadriparesis (10\%), and locked-in syndrome (5\%). Sex and cardiovascular risk factors (type 2 diabetes mellitus, hypercholesterolemia, and hypertension) or previous stroke or ischemic heart disease was not significantly associated with functional outcome.

The median GCS was 9 (IQR, 5-14). In 1 patient, the angiogram was not available for review; information on clot location, treatment, and recanalization was available from the procedural report, but TIMI and collaterals could not be assessed.

Clot location in the basilar artery (proximal, $n=12$; mid, $n=18$; or top-of-the-basilar, $n=5$ ) and presence of collaterals did not demonstrate any significant correlation with good neurologic outcome by Spearman analysis. Five patients had thrombus extending from the intradural VAs, of which 3 were bilateral. Nine had involvement of the extradural VAs, of which 2 were bilateral. In both the primary and secondary analyses, if thrombus extended from the VAs, $83 \%$ had a poor neurologic outcome. This is compared with $64 \%$ without VA involvement in patients with $\mathrm{mRS}>1$ and $57 \%$ in patients with $\mathrm{mRS}>2$.

Tables 1 and 2 reveal key clinical and angiographic data in those with 90 -day $m R S$ of $\leq 1$ and $>1$ and $m R S$ of $\leq 2$ and $>2$, respectively.

Median time to IA intervention was 7.2 hours (IQR, 5-12.5 hours). IA therapy included IA urokinase (95\%), mechanical thrombectomy with the Merci device (7.5\%), angioplasty $(35 \%)$, and stent insertion (17.5\%). Mean urokinase dose was 769,000 U. Mean procedural time to recanalization was $101 \pm$ 36.8 minutes. There was no correlation between mean procedural time and good neurologic outcome by the Student $t$ test. Recanalization occurred in $82.5 \%(n=33)$. TIMI 3 was achieved in 26 patients; TIMI 2 in 7; TIMI 1 in 4; and TIMI 0 in 2 patients. There were 3 intraoperative complications -2 ves- 


\begin{tabular}{|c|c|c|c|c|c|}
\hline & \multicolumn{2}{|c|}{$\mathrm{mRS} \leq 1(n=12)$} & \multicolumn{2}{|c|}{$\mathrm{mRS}>1(n=28)$} & \multirow[b]{2}{*}{$P$ Value } \\
\hline & No. & $\%$ & No. & $\%$ & \\
\hline$\overline{G C S}$ & & & & & 0.28 \\
\hline$\leq 6$ & 6 & 50.0 & 10 & 35.7 & \\
\hline$>6$ & 6 & 50.0 & 18 & 64.3 & \\
\hline Time to IA intervention & & & & & 0.07 \\
\hline$\leq 6$ Hours & 7 & 58.3 & 7 & 25.0 & \\
\hline$>6$ Hours & 5 & 41.7 & 21 & 75.0 & \\
\hline Recanalization & 11 & 91.7 & 22 & 78.6 & 0.65 \\
\hline Procedure time (mean) (min) & $95.9 \pm 13.0$ & & $103.5 \pm 7.2$ & & 0.59 \\
\hline TIMI score & & & & & 0.32 \\
\hline 0 & 1 & 8.3 & 1 & 3.6 & \\
\hline 1 & 0 & 0.0 & 4 & 14.3 & \\
\hline 2 & 1 & 8.3 & 6 & 21.4 & \\
\hline 3 & 10 & 83.3 & 16 & 57.1 & \\
\hline
\end{tabular}

\begin{tabular}{|c|c|c|c|c|c|}
\hline & \multicolumn{2}{|c|}{$\mathrm{mRS} \leq 2(n=14)$} & \multicolumn{2}{|c|}{$\mathrm{mRS}>2(n=26)$} & \multirow[b]{2}{*}{$P$ Value } \\
\hline & No. & $\%$ & No. & $\%$ & \\
\hline$\overline{G C S}$ & & & & & .79 \\
\hline$\leq 6$ & 6 & 42.9 & 10 & 38.5 & \\
\hline$>6$ & 8 & 57.1 & 16 & 61.5 & \\
\hline Time to IA intervention & & & & & .01 \\
\hline$\leq 6$ Hours & 9 & 64.3 & 5 & 19.2 & \\
\hline$>6$ Hours & 5 & 35.7 & 21 & 80.8 & \\
\hline Recanalization & 13 & 92.9 & 20 & 76.9 & .39 \\
\hline Procedure time (mean) (min) & $94.7 \pm 11.3$ & & $104.7 \pm 7.5$ & & .47 \\
\hline TIMI score & & & & & .44 \\
\hline 0 & 1 & 7.1 & 1 & 3.8 & \\
\hline 1 & 0 & 0.0 & 4 & 15.4 & \\
\hline 2 & 2 & 14.3 & 5 & 19.2 & \\
\hline 3 & 11 & 78.6 & 15 & 57.7 & \\
\hline
\end{tabular}

\begin{tabular}{|c|cc|c|c|c|c|}
\hline $\begin{array}{c}\text { mRS } 0 \\
1\end{array}$ & mRS 1 & mRS 2 & mRS 3 & mRS 4 & mRS 5 & mRS 6 \\
4
\end{tabular}

Fig 1. Distribution of $m R S$ for GCS $\leq 6$.

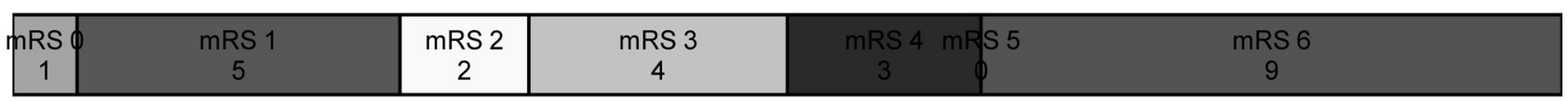

Fig 2. Distribution of $m R S$ for GCS $>6$.

sel perforations and 1 dissection. Five patients (12.5\%) had complicating intracranial hemorrhages; 4 of these patients died within 90 days. Overall, the 90-day mortality rate was $32.5 \%(n=13)$. Causes included intracranial hemorrhage $(n=4)$, extensive infarction or acute hydrocephalus $(n=3)$, failure to make neurologic recovery $(n=3)$, respiratory failure $(n=2)$, and retroperitoneal hematoma $(n=1)$.

Most outcome assessments were available at 3 months. Two mRS scores were taken at 2 months; 2 mRS scores, at 1 month; and $1 \mathrm{mRS}$ score, at 12 months. Figures 1 and 2 illustrate the distribution of $\mathrm{mRS}$ scores for patients with presenting GCS scores of $\leq 6$ and $>6$, respectively. Good neurologic outcome ( $\mathrm{mRS} \leq 1)$ occurred in 30\% (95\% CI, 16.6-46.5). Secondary analysis demonstrated good neurologic outcome $(\mathrm{mRS} \leq 2)$ in $35 \%$ of cases.

Stepwise logistic regression assessing GCS, age, recanalization, and time to IA intervention demonstrated that only time to IA intervention was a significant predictor of good neurologic outcome (with $\mathrm{mRS} \leq 2$ ). Those treated beyond 6 hours from symptom onset had less chance of good neurologic outcome $(\mathrm{mRS} \leq 2)$ (odds ratio $=0.106 ; 95 \% \mathrm{CI}, 0.023-0.488)$.

Subsequent analysis did not demonstrate any significant correlation between presenting GCS and mRS outcome (Spearman $\rho,-0.174 ; P=.283$ ). Equal numbers of patients with good neurologic outcome of $\mathrm{mRS} \leq 1(n=12)$ had a GCS of $\leq 6$ or $>6$.

Table 3 summarizes recanalization compared with mortality and mRS. Ninety-day mortality was $66.7 \%$ without recanalization (95\% CI, 22.3\%-95.7\%); if recanalization occurred, this improved to $27.2 \%$ (95\% CI, $13.3 \%-45.5 \% ; P=.15)$. If recanalization occurred, $33 \%$ had a good neurologic outcome of $\mathrm{mRS} \leq 1$, compared with $16 \%$ if recanalization did not occur $(P=.65)$. Of those who were recanalized, if time to IA intervention was $>6$ hours, $20 \%$ had a good neurologic out- 


\begin{tabular}{lccc}
\hline \multicolumn{3}{l}{ Table 3: Recanalization compared with } & 90-day mortality and mRS \\
\hline & $\begin{array}{c}\text { Failure of } \\
\text { Recanalization } \\
(n=6)\end{array}$ & $\begin{array}{c}\text { Recanalization } \\
(n=33)\end{array}$ & $\begin{array}{c}P \\
\text { Value }\end{array}$ \\
\hline $\begin{array}{c}\text { 90-Day mortality } \\
\text { 90-Day mRS } \\
\leq 1\end{array}$ & $4(67 \%)$ & $9(27 \%)$ & .15 \\
$>1$ & $1(17 \%)$ & $11(33 \%)$ & .65 \\
$\begin{array}{c}\text { 90-Day mRS } \\
\leq 2\end{array}$ & $5(83 \%)$ & $22(67 \%)$ & \\
$>2$ & $1(17 \%)$ & $13(39 \%)$ & \\
\hline
\end{tabular}

come. This improved to $54 \%$ if time to IA intervention was $\leq 6$ hours $(P=.07)$. In the secondary analysis, if recanalization occurred, $39 \%$ had a good neurologic outcome (mRS $\leq 2)$, compared with $17 \%$ if recanalization did not occur $(P=.391)$. Of those who were recanalized, if time to IA intervention was $>6$ hours, $20 \%$ had a good neurologic outcome (mRS $\leq 2$ ). This improved to $69 \%$ if time to IA intervention was $\leq 6$ hours $(P=.01)$.

\section{Discussion}

Early diagnosis and treatment are paramount in acute basilar artery thrombosis. While an acute onset of quadriplegia or locked-in state may be perceived as a common presentation, basilar artery thrombosis presents in a heterogeneous manner, and there should be a low threshold for investigation in the appropriate clinical setting. Ferbert et al ${ }^{19}$ studied 85 patients with angiographically proved basilar or bilateral distal vertebral occlusion, in whom sudden neurologic decline, including quadriplegia or locked-in state, occurred in only 20. Admission symptoms and signs were variable and included vertigo, headache, hemihyperesthesia, and oculomotor disturbance. Eleven percent were locked-in, and $18 \%$ were quadriplegic ${ }^{19}$ compared with 5\% locked-in and 5\% quadriplegic in our study.

The natural history of acute basilar artery thrombosis managed with antiplatelets and/or anticoagulants is poor. Hacke et $\mathrm{al}^{2}$ reported 19 of 22 deaths $(86 \%)$ in a cohort of patients who received antiplatelet or anticoagulant therapy, while Schonewille et $\mathrm{al}^{20}$ described dependency rates ( $\mathrm{mRS} 4-5$ ) of $\leq 65 \%$ and mortality rates of $40 \%$ in a similar subgroup of patients. The spontaneous recanalization rate in acute basilar artery thrombosis is unknown but is suggested to be no higher than $20 \%$ within $12-24$ hours. ${ }^{6}$ This compares with IA thrombolysis that can achieve recanalization rates as high as $92 \% .{ }^{6,11}$ The recanalization rate in our study population was comparable at $82.5 \%$.

Recanalization is associated with a significant survival benefit, particularly when data from multiple studies are included. ${ }^{6}$ Seventy-three percent (95\% CI, 54.5\%-86.8\%) of those who were recanalized survived at 90 days, compared with 33\% (95\% CI, 4.3\%-77.7\%) of those who were not recanalized $(P=.15)$. A larger sample size may have resulted in a significant survival benefit, but in the examination of 83 patients by in Eckert et al, ${ }^{5}$ mortality reduction from $72 \%$ to $54 \%$ with recanalization was not significant. Ten of the 14 recanalized patients examined by Hacke et $\mathrm{al}^{2}$ had a favorable neurologic outcome (defined as no, minimal, or moderate neurologic deficit). In our study population, 33\% of those recanalized had a good neurologic outcome of $\mathrm{mRS} \leq 1$, compared with $16 \%$ if recanalization did not occur. Our definition is stricter, with $\mathrm{mRS} \leq 1$ (ie, no symptoms at all, no significant disability despite symptoms, and able to carry out all usual duties and activities). This is not dissimilar to the study of Eckert et al, in which $30 \%$ of those who were recanalized had a 3-month Barthel index of $>90$, compared with $10 \%$ of those who were not recanalized. Of our 6 patients who were unable to be recanalized (TIMI, 0 or 1 ) and were managed medically, 4 died and 1 had severe disability (mRS 5).

Of those who were recanalized, there was a trend toward more frequent good neurologic outcome ( $\mathrm{mRS} \leq 1)$ with earlier treatment $(20 \%$ if treated $>6$ hours compared with $54 \%$ if treated $\leq 6$ hours, $P=.07$ ). In the secondary analysis ( $\mathrm{mRS} \leq$ 2 as a good neurologic outcome), statistical significance was demonstrated between time to IA intervention and good outcome, increasing from $20 \%$ if it was $>6$ hours to $69 \%$ if, within 6 hours $(P=.01)$. These findings add strength to the study of Eckert et $\mathrm{al}^{5}$ in which, with just over double the sample size, a significant improvement in favorable neurologic outcome was reported (Barthel index, >90) from $7 \%$ to $36 \%$ if treatment could be performed within 6 hours. Eckert et al subsequently concluded that endovascular treatment is not reasonable if patients present with a persistent severe neurologic deficit like tetraplegia and coma for $>6$ hours.

There was no correlation with GCS and 90-day mRS. Of the 12 patients with a mRS $\leq 1$, six had GCS $\leq 6$, the same number as those with GCS $>6$.

Although time to IA intervention may be a confounding factor in this assessment, of the 15 patients with GCS $\leq 6$ for $>3$ hours, a third had good neurologic outcome ( $\mathrm{mRS} \leq 1)$ at 90 days. This is a rate to similar that of the overall cohort of 40 , with $30 \%$ good neurologic outcome overall. There were 10 patients with GCS of $\leq 6$ and $>6$ hours to IA intervention. Three had an mRS score of 5 , and 4 were dead at 90 days. However, notable cases include a 79-year-old patient with GCS 6 treated at 6 hours with a 90-day mRS of 1 . An 82-yearold patient with a GCS of 6 who was treated at 12 hours made a full neurologic recovery with an $\mathrm{mRS}$ of 0 at 3-month review. A 63-year-old patient with a GCS score of 3 for $>3$ hours was treated at 5 hours, with a 90-day mRS of 1 after IA lysis.

There are weaknesses in our study; it was a retrospective case review with a small sample size. Similar to treatment in other stroke referral centers, preoperative anticoagulation was variable, particularly when referred from external institutions. Clinical follow-up was not fully standardized, with 4 of 40 patient outcomes measured at 1-2 months (mRS 3, $n=2$; mRS $4, n=1$; mRS $5, n=1$ ) and 1 patient outcome recorded at 12 months ( $\mathrm{mRS} 4$ ) postintervention. Variations in the timing of mRS scores were subsequent to patients being lost to follow-up; however, their outcome data were included to minimize biasing of the population outcome. For the remainder, patient functional outcomes demonstrated ongoing improvement or stability, with increasing time postintervention, with only 1 case presenting with stent reocclusion 3 months following discharge, in the context of subtherapeutic warfarin therapy. This result suggests that our outcome data may be a conservative estimate of good neurologic outcome. The 16-year study period has also seen significant improvement in diagnostic angiography suites, neurointerventional catheters and 
wires, and new mechanical thrombectomy devices. Nonetheless, it represents 16 years of consecutive angiographically proved basilar thromboses in routine clinical comprehensive stroke care settings.

\section{Conclusions}

GCS is frequently used as a selection criterion for IA therapy in acute basilar artery thrombosis based on the view that higher GCS correlates with better neurologic outcome. However, when systematically studied, there was no correlation between GCS and neurologic outcome at 90 days. Thus, we propose that it is not appropriate to exclude patients from IA therapy on the basis of low GCS scores. Continuing data collection by our neurointerventional group will provide further data to confirm our current findings on a larger sample population.

\section{Acknowledgments}

We thank Alexandra Gorelik, a statistician from the Clinical Epidemiology and Health Service Evaluation Unit (The Royal Melbourne Hospital), for her statistical analysis and advice during preparation of the manuscript.

\section{References}

1. Archer CR, Horenstein S. Basilar artery occlusion: clinical and radiological correlation. Stroke 1977;8:383-90

2. Hacke W, Zeumer H, Ferbert A, et al. Intra-arterial thrombolytic therapy improves outcome in patients with acute vertebrobasilar occlusive disease. Stroke 1988;19:1216-22

3. Brandt T, von Kummer R, Muller-Kuppers M, et al. Thrombolytic therapy of acute basilar artery occlusion: variables affecting recanalization and outcome. Stroke 1996;27:875-81

4. Pfefferkorn T, Mayer TE, Opherk C, et al. Staged escalation therapy in acute basilar artery occlusion: intravenous thrombolysis and on-demand consecutive endovascular mechanical thrombectomy-preliminary experience in $\mathbf{1 6}$ patients. Stroke 2008;39:1496-500

5. Eckert B, Kucinski T, Pfeiffer G, et al. Endovascular therapy of acute vertebro- basilar occlusion: early treatment onset as the most important factor. Cerebrovasc Dis 2002;14:42-50

6. Smith WS. Intra-arterial thrombolytic therapy for acute basilar occlusion: pro. Stroke 2007;38:701-03

7. Lindsberg PJ, Mattle HP. Therapy of basilar artery occlusion: a systematic analysis comparing intra-arterial and intravenous thrombolysis. Stroke 2006;37:922-28

8. Mitchell PJ, Gerraty RP, Donnan GA, et al. Thrombolysis in the vertebrobasilar circulation: the Australian Urokinase Stroke Trial—a pilot study. Cerebrovasc Dis 1997;7:94-99

9. Macleod MR, Davis SM, Mitchell PJ, et al. Results of a multicentre, randomised controlled trial of intra-arterial urokinase in the treatment of acute posterior circulation ischaemic stroke. Cerebrovasc Dis 2005;20:12-17. Epub 2005 May 30

10. Schulte-Altedorneburg G, Brückmann H, Hamann GF, et al. Ischemic and hemorrhagic complications after intra-arterial fibrinolysis in vertebrobasilar occlusion. AJNR Am J Neuroradiol 2007;28:378-81

11. Nagel S, Schellinger PD, Hartmann M, et al. Therapy of acute basilar artery occlusion: intraarterial thrombolysis alone vs bridging therapy. Stroke 2009:40:140-46

12. Arnold M, Nedeltchev K, Schroth G, et al. Clinical and radiological predictors of recanalisation and outcome of 40 patients with acute basilar artery occlusion treated with intra-arterial thrombolysis. J Neurol Neurosurg Psychiatry 2004; $75: 857-62$

13. Schellinger PD, Hacke W. Intra-arterial thrombolysis is the treatment of choice for basilar thrombosis: pro. Stroke 2006;37:2436-37

14. Schulte-Altedorneburg G, Hamann GF, Mull M, et al. Outcome of acute vertebrobasilar occlusions treated with intra-arterial fibrinolysis in 180 patients. AJNR Am J Neuroradiol 2006;27:2042-47

15. Brandt T. Diagnosis and thrombolytic therapy of acute basilar artery occlusion: a review. Clin Exp Hypertens 2002;24:611-22

16. Ezaki Y, Tsutsumi K, Onizuka M, et al. Retrospective analysis of neurological outcome after intra-arterial thrombolysis in basilar artery occlusion. Surg Neurol 2003;60:423-30

17. Tsao JW, Hemphill JC, Johnston SC, et al. Initial Glasgow Coma Scale score predicts outcome following thrombolysis for posterior circulation stroke. Arch Neurol 2005;62:1126-29

18. Demchuk AM, Tanne D, Hill MD, et al. Predictors of good outcome after intravenous tPA for acute ischemic stroke. Neurology 2001;57:474-80

19. Ferbert A, Brückmann H, Drummen R. Clinical features of proven basilar artery occlusion. Stroke 1990;21:1135-42

20. Schonewille WJ, Algra A, Serena J, et al. Outcome in patients with basilar artery occlusion treated conventionally. J Neurol Neurosurg Psychiatry 2005;76: $1238-41$ 